\title{
Renormalization of the baryon axial vector current in large- $N_{c}$
}

\author{
María A. Hernández-Ruiz ${ }^{\dagger}$ \\ Instituto de Física, Universidad Autónoma de San Luis Potosí \\ Av. Manuel Nava 6 Zona Universitaria 78290, San Luis Potosí SLP, México. \\ Facultad de Ciencias Químicas Universidad Autónoma de Zacatecas \\ Apartado Postal 585, 98060 Zacatecas, México. \\ mahernandifisica.uaslp.my
}

\begin{abstract}
The baryon axial vector current is computed at one-loop order in heavy baryon chiral perturbation theory in the large $-N_{c}$ limit, where $N_{c}$ is the number of colors. Loop graphs with octet and decuplet intermediate states cancel to various orders in $N_{c}$ as a consequence of the large- $N_{c}$ spinflavor symmetry of QCD baryons. We present a preliminary study of the convergence of the chiral expansion with $1 / N_{c}$ corrections. The physical values $N_{f}=3$ (where $N_{f}$ is the number of light quark flavors) and $N_{c}=3$ are used in the case of $g_{A}$ in QCD.
\end{abstract}

35th International Conference of High Energy Physics - ICHEP2010,

July 22-28, 2010

Paris France

\footnotetext{
*Speaker.

${ }^{\dagger}$ The author would like to express their gratitude to Local Organizing Committee of the 35th International Conference of High Energy Physics also acknowledge support.
} 


\section{Renormalization of the baryon axial vector current}

The QCD operators have well-defined $1 / N_{c}$ expansions. For the baryon axial vector current $A^{k c}$ its $1 / N_{c}$ expansion can be written as

$$
A^{k c}=a_{1} G^{k c}+\sum_{n=2,3}^{N_{c}} b_{n} \frac{1}{N_{c}^{n-1}} \mathscr{D}_{n}^{k c}+\sum_{3,5}^{N_{c}} c_{n} \frac{1}{N_{c}^{n-1}} \mathscr{O}_{n}^{k c},
$$

The correction containing the full dependence on the ratio $\Delta / m_{\Pi}$, where $\Delta \equiv M_{\Delta}-M_{N}$ is the decuplet-octet mass difference and $m_{\Pi}$ is the meson mass. Was derived in Ref. [四] and reads

$$
\begin{aligned}
\delta A^{k c} & =\frac{1}{2}\left[A^{j a},\left[A^{j b}, A^{k c}\right]\right] \Pi_{(1)}^{a b}-\frac{1}{2}\left\{A^{j a},\left[A^{k c},\left[\mathscr{M}, A^{j b}\right]\right]\right\} \Pi_{(2)}^{a b} \\
& +\frac{1}{6}\left(\left[A^{j a},\left[\left[\mathscr{M},\left[\mathscr{M}, A^{j b}\right]\right], A^{k c}\right]\right]-\frac{1}{2}\left[\left[\mathscr{M}, A^{j a}\right],\left[\left[\mathscr{M}, A^{j b}\right], A^{k c}\right]\right]\right) \Pi_{(3)}^{a b}+\ldots
\end{aligned}
$$

Here $\Pi_{(n)}^{a b}$ is a symmetric tensor which contains meson-loop integrals with the exchange of a single meson: A meson of flavor $a$ is emitted and a meson of flavor $b$ is reabsorbed. $\Pi_{(n)}^{a b}$ descomposes into flavor singlet, flavor 8 and flavor 27 representations []].

\section{Results and Conclusions}

We have computed the renormalization of the baryon axial vector current in the framework of heavy baryon chiral perturbation theory in the large $-N_{c}$ limit. The matrix elements of the space components of $A^{k c}$ between $S U(6)$ symetric states give the actual values of the axial vector couplings. For $N_{c}=3$ and $N_{f}=3$ the couplings $g_{A}$ of barions with corrections at relative orders $N_{c}, 1 / N_{c}, 1 / N_{c}^{2}$ and $1 / N_{c}^{3}$ for flavor singlet contribution are shown in Table 1.

\begin{tabular}{lrrrrr}
\hline \hline \multicolumn{5}{c}{ Singlet } & \\
\hline Process & $\mathscr{O} N_{c}$ & $\mathscr{O}\left(1 / N_{c}\right)$ & $\mathscr{O}\left(1 / N_{c}^{2}\right)$ & $\mathscr{O}\left(1 / N_{c}^{3}\right)$ & Total \\
\hline$n \rightarrow p e^{-} \bar{v}_{e}$ & 1.271 & -0.1138 & 0.1402 & -0.0256 & 1.272 \\
$\Sigma^{+} \rightarrow \Lambda e^{+} v_{e}$ & 0.615 & -0.0396 & 0.0663 & 0.0111 & 0.653 \\
$\Sigma^{-} \rightarrow \Lambda e^{-} \bar{v}_{e}$ & 0.598 & -0.0266 & 0.0446 & 0.0074 & 0.624 \\
$\Lambda \rightarrow p e^{-} \bar{v}_{e}$ & -0.941 & 0.0837 & -0.0855 & 0.0389 & -0.904 \\
$\Sigma^{-} \rightarrow n e^{-} \bar{v}_{e}$ & 0.330 & 0.0014 & 0.0188 & 0.0239 & 0.375 \\
$\Xi^{-} \rightarrow \Lambda e^{-} \bar{v}_{e}$ & 0.212 & -0.0423 & 0.0179 & -0.0483 & 0.139 \\
$\Xi^{-} \rightarrow \Sigma^{0} e^{-} \bar{v}_{e}$ & 0.868 & -0.0522 & 0.0643 & -0.0117 & 0.869 \\
$\Xi^{0} \rightarrow \Sigma^{+} e^{-} \bar{v}_{e}$ & 1.310 & -0.0998 & 0.1231 & -0.0225 & 1.312 \\
\hline \hline
\end{tabular}

Table 1: Corrections at relative order for the axial vector couplings of the baryons

\section{References}

[1] R. Flores-Mendieta, C. P. Hofmann, E. Jenkins, and A. V. Manohar, Phys. Rev. D 62, 034001 (2000).

[2] R. Flores-Mendieta, C. P. Hofmann, Phys. Rev. D 74, 094001 (2006). 